\title{
Power law correlations in galaxy distribution and finite volume effects from the Sloan Digital Sky Survey Data Release Four
}

\author{
F. Sylos Labini ${ }^{1,2}$, N. L. Vasilyev ${ }^{3}$, and Y. V. Baryshev ${ }^{3}$ \\ 1 "Enrico Fermi Center", via Panisperna 89 A, Compendio del Viminale, 00184 Rome, Italy \\ e-mail: sylos@romal.infn.it \\ 2 "Istituto dei Sistemi Complessi" CNR, via dei Taurini 19, 00185 Rome, Italy \\ ${ }^{3}$ Institute of Astronomy, St. Petersburg State University, Staryj Peterhoff, 198504 St. Petersburg, Russia
}

Received 30 March 2006 / Accepted 18 October 2006

ABSTRACT

\begin{abstract}
We discuss the estimation of galaxy correlation properties in several volume limited samples, in different sky regions, obtained from the Fourth Data Release of the Sloan Digital Sky Survey. The small scale properties are characterized through the determination of the nearest neighbor probability distribution. By using a very conservative statistical analysis, in the range of scales $[0.5, \sim 30] \mathrm{Mpc} / h$ we detect power-law correlations in the conditional density in redshift space, with an exponent $\gamma=1.0 \pm 0.1$. This behavior is stable in all the different samples we considered; thus it does not depend on galaxy luminosity. In the range of scales $[\sim 30, \sim 100] \mathrm{Mpc} / h$ we find evidence for systematic unaveraged fluctuations and we discuss in detail the problems induced by finite volume effects on the determination of the conditional density. We conclude that in such a range of scales there is evidence for a smaller power-law index of the conditional density. However we cannot distinguish between two possibilities: (i) that a crossover to homogeneity (corresponding to $\gamma=0$ in the conditional density) occurs before $100 \mathrm{Mpc} / h$; (ii) that correlations extend to scales of order $100 \mathrm{Mpc} / h$ (with a smaller exponent $0<\gamma<1$ ). We emphasize that galaxy distributions in these samples present large fluctuations at the largest scales probed, corresponding to the presence of large scale structures extending up to the boundaries of the present survey. We discuss several differences between the behavior of the conditional density in mock galaxy catalogs built from cosmological $N$-body simulations and real data. We discuss some theoretical implications of such differences considering also the super-homogeneous features of primordial density fields.
\end{abstract}

Key words. cosmology: observations - large-scale structure of Universe

\section{Introduction}

A major problem in modern cosmology is the statistical characterization and the physical understanding of large scale galaxy structures. The first question in this context concerns the studies of galaxy correlation properties. Two-point properties are particularly useful to determine correlations and their spatial extension. There are different ways of measuring two-point properties and, in general, the most suitable method depends on the type of correlation, strong or weak, characterizing a given point distribution in a sample.

For example, Hogg et al. (2005) recently measured the conditional average density in a sample of Luminous Red Galaxies (LRG) from a data release of the Sloan Digital Sky Survey (SDSS). Such a statistic is very useful to determine correlation properties in the regime of strong clustering and the spatial extension of strong fluctuations in a given sample. This was firstly introduced by Pietronero (1987) and then measured in many samples by Sylos Labini et al. (1998). We refer the reader to Baryshev \& Teerikorpi (2006) for a review of the measurements of the reduced and complete correlation functions by different authors in the various angular and three-dimensional samples.

The conditional density gives the average density of points in a spherical volume (or a spherical shell) centered around a galaxy (see Gabrielli et al. 2004, for a discussion of this method). The results obtained by Hogg et al. (2005) can be summarized as follows:

(i) A simple power-law scaling corresponding to a correlation exponent $\gamma \approx 1$ gives a very good fit to the data up to at least $20 \mathrm{Mpc} / h$, over approximately a decade in scale. These results are in good agreement with those obtained by Sylos Labini et al. (1998) through the analyses of many smaller samples and more recently by Vasilyev et al. (2006) in the 2dFGRS.

(ii) At larger scales (i.e. $r>30 \mathrm{Mpc} / h$ ) the conditional density continues to decrease, but less rapidly, until about $\sim 70 \mathrm{Mpc} / h$, above which it seems to flatten up to the largest scale probed by the sample $(100 \mathrm{Mpc} / h)$. The transition between the two regimes is slow, in the sense that the conditional density at $\sim 20 \mathrm{Mpc} / h$ is about twice the asymptotic mean density. Joyce et al. (2005) have discussed the implications of these results noticing, for example, that the possible convergence to a well defined homogeneity in a volume equivalent to that of a sphere of radius $70 \mathrm{Mpc} / \mathrm{h}$, places in doubt previous detections of "luminosity bias" from measures of the amplitude of the reduced correlation function $\xi(r)$. They emphasized that the way to resolve these issues is, in volume limited (VL) samples corresponding to different ranges of luminosity, to first use the conditional density to establish the features of galaxy space correlations. Sylos Labini et al. (1998) found evidence for a continuation 
of the small scale power-law to distances of the order of hundreds of $\mathrm{Mpc} / h$, although with weaker statistics, which seems not be confirmed by Hogg et al. (2005).

In this paper we continue the analysis of galaxy distributions previously applied to the $2 \mathrm{dFGRS}$ data (Vasilyev et al. 2006) to the so-called "main galaxy sample" of SDSS Data Release (DR4), in the spirit of the tests discussed above. In a companion paper we discuss the properties of the LRG sample of the SDSS DR4, which can be directly compared with the results of Hogg et al. (2005) and Eiseinstein et al. (2005).

The paper is organized as follows. In Sect. 2 we describe the data and the way we have constructed the VL samples. We also discuss the determination of the nearest neighbor (NN) distribution, and of the average distance between nearest galaxies, which allows us to define the lower cut-off for the studies of correlations. In addition we discuss the determination of the radial counts in different VL samples, emphasizing that large variations for this quantity are found in the different samples. Such fluctuations, which seem to be persistent up to the sample boundaries, correspond to the large scale structures observed in these catalogs. The quantitative characterization of the correlation properties of these fluctuations is presented in Sect. 3, where we discuss the determination of the conditional average density in the different VL samples. In particular we present several tests useful to clarify the effect of systematic fluctuations at scales of the order of the sample size.

In Sect. 4 we discuss the differences between the galaxy conditional density, measured in these samples and the conditional density of point-particles in cosmological $N$-body simulations. We show that by using these statistics, together with a study of the NN probability distribution, two-point properties of observed galaxies of different luminosity and mock galaxy catalogs constructed using particles lying in region with different local density in cosmological $N$-body simulations, present different behaviors. In Sect. 5 we draw our main conclusions.

\section{The data}

The SDSS (http://www.sdss.org) is currently the largest spectroscopic survey of extragalactic objects and one of the most ambitious observational programs ever undertaken in astronomy. It will measure about 1 million redshifts, giving a complete mapping of the local universe up to a depth of several hundreds of Mpc. In this paper we consider the data from the latest public data release (SDSS DR4) which is accessible at http://www.sdss.org/dr4 (Adelman-McCarthy et al. 2005) containing redshifts for more than 565 thousand galaxies and 67 thousand quasars. There are two independent parts of the galaxy survey in the SDSS: the main galaxy sample and the LRG sample. Here we discuss the former only. The spectroscopic survey covers an area of 4783 square degrees of the celestial sphere. The apparent magnitude limit for the galaxies is 17.77 in the $r$-filter and photometry for each galaxy is available in five different bands, of which we consider the ones in the $r$ and $g$ filters.

\subsection{Definition of the samples}

We have used the following criteria to query the SDSS DR4 database. We constrain the flags indicating the type of object so that we select only the objects from the main galaxy sample. We then consider galaxies in the redshift interval $10^{-4} \leq z \leq 0.3$ and
Table 1. Main properties of the angular regions considered: The limits in degrees of the cuts are chosen using the intrinsic coordinates of the survey $\eta$ and $\lambda$ (in degrees). The last column $\Omega$ gives the solid angle of three angular regions in steradians.

\begin{tabular}{cccccc}
\hline \hline Region name & $\eta_{1}$ & $\eta_{2}$ & $\lambda_{1}$ & $\lambda_{2}$ & $\Omega$ \\
\hline R1 & 9.0 & 36.0 & -47.0 & 8.0 & 0.41 \\
R2 & -33.5 & -16.5 & -54.0 & -24.0 & 0.12 \\
R3 & -36.0 & -26.5 & 2.5 & 43.0 & 0.11 \\
\hline
\end{tabular}

with the redshift confidence parameter larger than 0.95 . In addition we apply the filtering condition $r<17.77$, thus taking into account the target magnitude limit for the main galaxy sample in the SDSS DR4. Thus we have selected 321516 objects.

The angular coverage of the survey is not uniform but observations have been done in different sky regions. For this reason we have considered three rectangular angular fields (named R1, $\mathrm{R} 2$ and R3) in the SDSS internal angular coordinates $(\eta, \lambda)$ : in such a way we do not have to consider the irregular boundaries of the survey mask, as we have cut such boundaries to avoid uneven edges of observed regions. In Table 1 we report the parameters of the three angular regions considered. We do not use corrections for the redshift completeness mask or for fiber collision effects. Completeness varies most near the current survey edges which are excluded in our samples. Fiber collisions in general do not present a problem for measurements of galaxy correlations (see discussion in, e.g., Strauss et al. 2002).

\subsection{Construction of VL samples}

To construct VL samples that are unbiased for the selection effect related to the cut in the apparent magnitude, we have applied a standard procedure (see e.g. Zehavi et al. 2004): we compute metric distances as

$r(z)=\frac{c}{H_{0}} \int_{\frac{1}{1+z}}^{1} \frac{\mathrm{d} y}{y \cdot\left(\Omega_{\mathrm{M}} / y+\Omega_{\Lambda} \cdot y^{2}\right)^{1 / 2}}$,

where we have used the standard cosmological parameters $\Omega_{\mathrm{M}}=$ 0.3 and $\Omega_{\Lambda}=0.7$ with $H_{0}=100 h \mathrm{~km} \mathrm{~s}^{-1} \mathrm{Mpc}^{-1}$.

We use Petrosian apparent magnitudes in the $r$ filter $m_{r}$ which are corrected for galactic absorption. The absolute magnitudes can be computed as

$M_{r}=m_{r}-5 \cdot \log _{10}[r(z) \cdot(1+z)]-K_{r}(z)-25$,

where $K_{r}(z)$ is the K-correction. As the redshift range considered is small from a cosmological point of view (i.e. $z \leq 0.3$ ), to estimate the K-corrections $K_{r}(z)$ (linearly proportional to $z$ and thus small in this context) we have used the simple interpolating formula

$K_{r}(z)=\left(2.61 \cdot\left(m_{g}-m_{r}\right)-0.64\right) \cdot z$

where $m_{g}$ is the apparent magnitude in the $g$ filter. This corresponds to the calculated K-corrections in Blanton et al. (2001 see their Fig. 4). By knowing the intrinsic $g-r$ color and the redshift one directly estimates the $K$-correction term.

We have considered 4 different VL samples (named VL1, VL2, VL3 and VL4) defined by two chosen limits in absolute magnitude and metric distance, whose parameters are reported in Table 2. While VL1 and VL2 contain relatively faint galaxies in the local universe, the VL3 sample covers a wide range of distances, and VL4 consists of bright galaxies at distances up to $600 \mathrm{Mpc} / h$. Considering the three different rectangular areas 
Table 2. Main properties of the obtained VL samples: $r_{\min }, r_{\max }$ (in $\mathrm{Mpc} / h$ ) are the chosen limits for the metric distance; $M_{\min }, M_{\max }$ define the interval for the absolute magnitude in each sample. The quantity $\langle\Lambda\rangle$ (in $\mathrm{Mpc} / h)$ is the average distance between nearest-neighbor galaxies.

\begin{tabular}{cccccc}
\hline \hline VL sample & $r_{\min }$ & $r_{\max }$ & $M_{\min }$ & $M_{\max }$ & $\langle\Lambda\rangle$ \\
\hline VL1 & 50 & 135 & -19.0 & -18.0 & 1.7 \\
VL2 & 50 & 200 & -21.0 & -19.0 & 1.3 \\
VL3 & 100 & 500 & -23.0 & -21.0 & 2.9 \\
VL4 & 150 & 600 & -23.0 & -22.0 & 6 \\
\hline
\end{tabular}

Table 3. Number of galaxies in each of the VL samples. Names are given according to the discussion in the text. The scale $R_{\mathrm{c}}$ (in $\mathrm{Mpc} / h$ ) is discussed in Sect. 3.2 below.

\begin{tabular}{lcc}
\hline \hline VL Sample & $N$ & $R_{\mathrm{c}}$ \\
\hline R1VL1 & 3130 & 15 \\
R1VL2 & 15181 & 21 \\
R1VL3 & 27975 & 54 \\
R1VL4 & 6742 & 65 \\
R2VL1 & 790 & 10 \\
R2VL2 & 3912 & 15 \\
R2VL3 & 8586 & 38 \\
R2VL4 & 1923 & 42 \\
R3VL1 & 790 & 9 \\
R3VL2 & 2895 & 12 \\
R3VL3 & 7584 & 30 \\
R3VL4 & 1503 & 36 \\
\hline
\end{tabular}

(described above), we have $4 \times 3=12 \mathrm{VL}$ subsamples, whose characteristics are reported in Table 3 . The comparison between VL samples with the same magnitude and distance cuts, in different sky regions, will allow us to test the statistical stationarity of galaxy distributions in these samples and to estimate sampleto-sample fluctuations.

\subsection{Nearest neighbor distribution}

The NN distance probability distribution depends on the cut in absolute magnitude of a given VL sample. We expect this function not to be dependent on the angular sky cuts if the distribution is statistically stationary in the different VL samples. As discussed in Vasilyev et al. (2006) space correlations introduce a deviation from the case of a pure Poisson distribution: the average distance $\langle\Lambda\rangle$ between NN is expected to be smaller than for the Poisson case in the same sample and with the same number of points. The measurements in the data, obtained by simple paircounting, are shown in Figs. 1-4. When a VL sample includes fainter galaxies (e.g. VL1, VL2) $\langle\Lambda\rangle$ is smaller (see Table 2) than for the case when only brighter galaxies are inside (e.g. VL3, VL4). This is because brighter galaxies are sparser than fainter ones. This corresponds to the exponential decay of the galaxy luminosity function at the bright end (see discussion in Gabrielli et al. 2004)

Zehavi et al. (2004) have estimated that at a scale of the order of $1 \div 2 \mathrm{Mpc} / h$ there is a departure from a power law behavior in the reduced correlation function. In the light of the discussion above we stress that this change occurs over a range of scales where NN correlations are dominant in all samples considered. For the interpretation of this behavior one may consider the relation between the conditional density, or the reduced correlation function, and the NN probability distribution (see Baertschiger \& Sylos Labini 2004, for a discussion of this point). In this

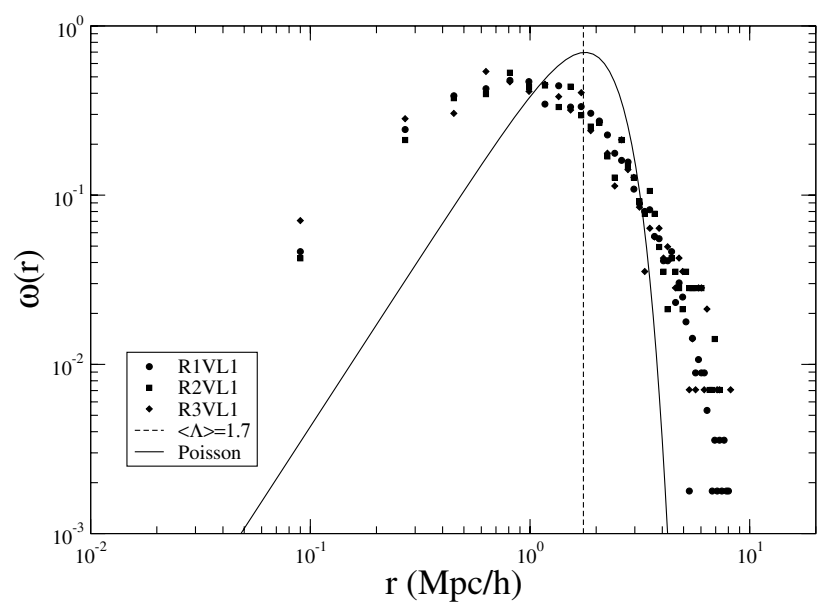

Fig. 1. Nearest Neighbor distribution in the VL1 sample: different symbols correspond to different angular regions. The average distance between nearest galaxies is $\langle\Lambda\rangle=1.7 \mathrm{Mpc} / h$. For reference the solid line represents the $\mathrm{NN}$ distribution for a Poisson configuration with the same $\langle\Lambda\rangle$ : the tails of this function decay more rapidly.

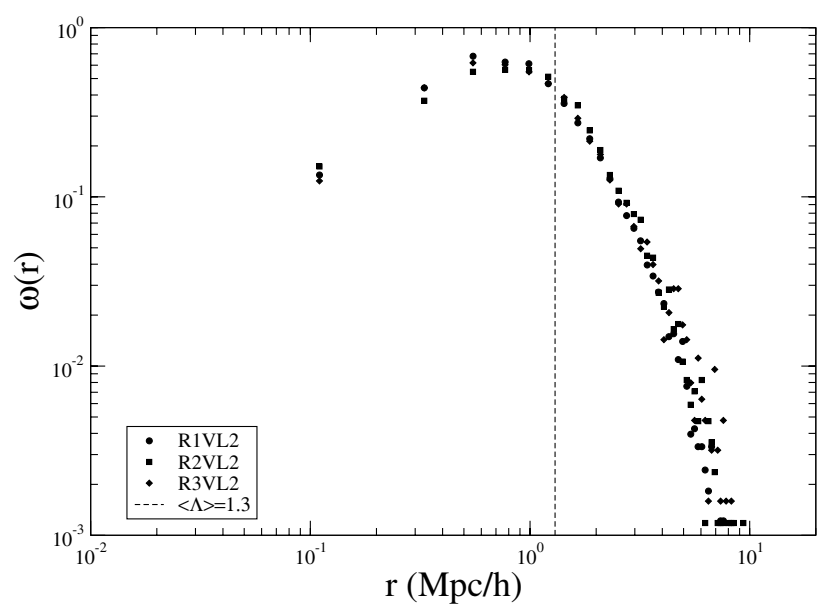

Fig. 2. As Fig. 1 but for the VL2 samples. The average distance between galaxies is $\langle\Lambda\rangle=1.3 \mathrm{Mpc} / h$.

respect, in the comparison of galaxy data with $\mathrm{N}$-body simulations, one has to be careful in that these small-scale properties can be determined by sampling, sparseness and other more subtle finite size effects related to the precision of a given $N$-body simulation (Baertschiger \& Sylos Labini 2004).

We have then studied the effect of the fiber collisions on the NN statistic: about $6 \%$ of galaxies that satisfy the selection criteria of the main galaxy sample are not observed because they have a companion closer than the 55 arcsec minimum separation of spectroscopic fibers (Strauss et al. 2002). However not all 55-arcsec pairs are affected by fiber collisions, because some of the SDSS were observed spectroscopically more than once. We have identified all $<=55$ arcsec pairs for which both galaxies have redshifts, and we have randomly removed one of those redshifts in each case to make a new sample with an even more severe fiber collision problem than the existing sample. Because of the very small number of galaxy pairs with angular separation $<=55$ arcsec (of the order of a few percent in all the volume limited samples we have considered) there is no noticeable effect of the results. For galaxies in the main sample the average redshift $z \sim 0.1$, and hence the angular distance 55 arcsec corresponds to the linear separation $r \sim 0.1 \mathrm{Mpc} / h$ which is marginally outside 


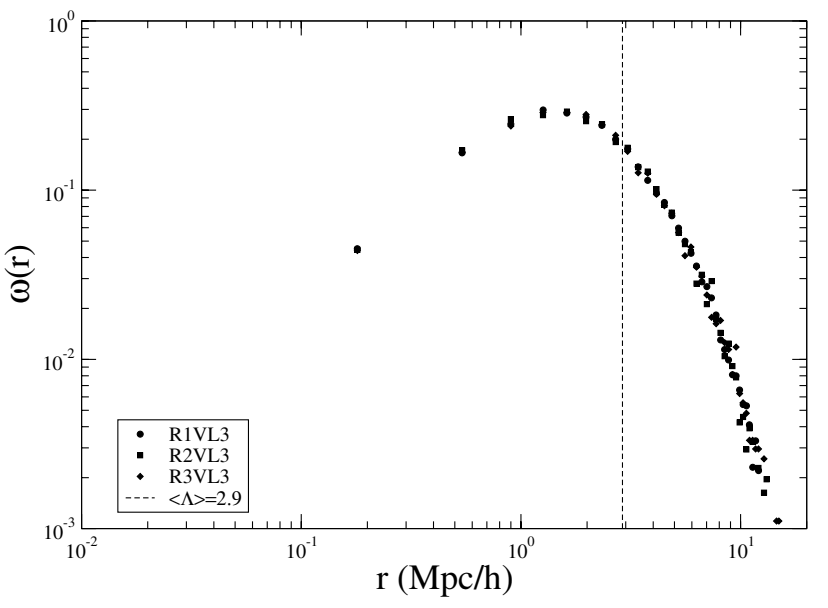

Fig. 3. As Fig. 1 but for the VL3 samples. The average distance between nearest galaxies is $\langle\Lambda\rangle=2.9 \mathrm{Mpc} / h$.

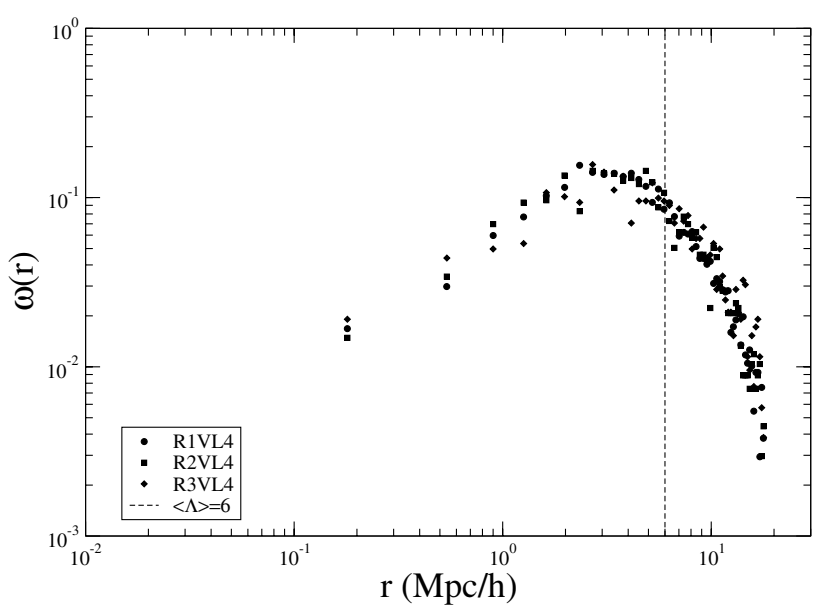

Fig. 4. As Fig. 1 but for the VL4 samples. The average distance between nearest galaxies is $\langle\Lambda\rangle=6 \mathrm{Mpc} / h$.

the scale interval in which we have studied the $\mathrm{NN}$ distribution, i.e. $r>0.2 \mathrm{Mpc} / h$. Hence we expect that the fiber collision effect does not influence our results as indeed we find.

\subsection{Number counts in VL samples}

A simple statistic a value of that can be easily computed in VL samples is the differential number counts. This gives us a first indication about (i) the slope of the counts; and (ii) the nature of fluctuations (see e.g. Gabrielli et al. 2004). In general we may write that the number of points counted from a given point chosen as the origin (in this case the Earth) grows as

$N(r) \sim r^{D}$.

This represents the radial counts in a spherical volume of radius $r$ around the observer (or in a portion of a sphere). In the case $D=3$ the distribution is uniform and $D<3$ if it is, for example, fractal or if there is a systematic effect of depletion of points as a function of distance. In this situation we neglect relativistic effects, which are small in the range of redshift considered. However, as noticed by Gabrielli et al. (2004) these corrections may change the slope of the counts but not the intrinsic fluctuations.

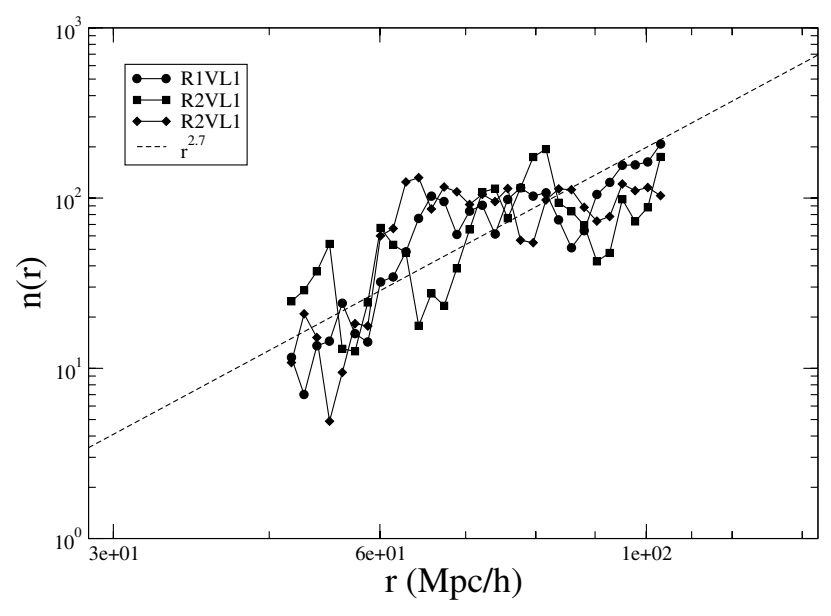

Fig. 5. Differential number counts as a function of distance in the VL1 sample in different angular regions normalized to their own solid angle.

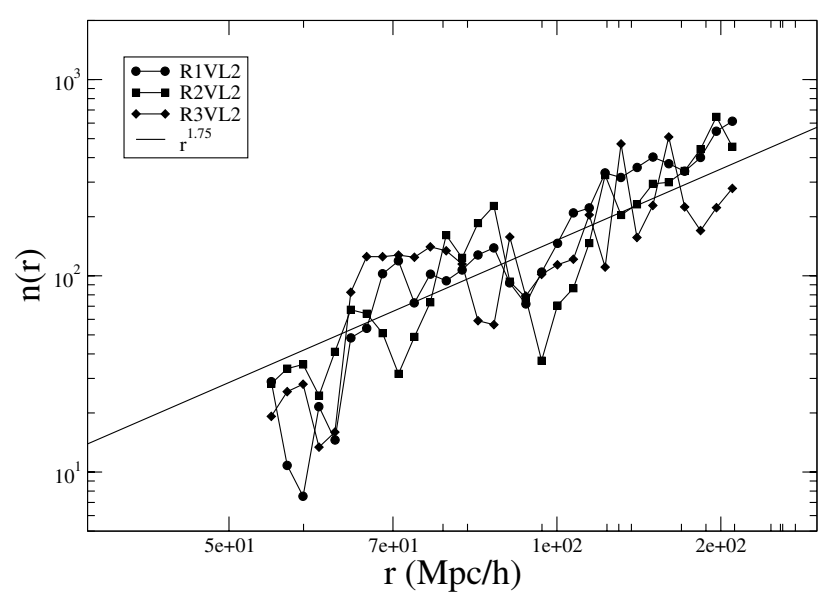

Fig. 6. The same as Fig. 5 but for the VL2 samples.

Given that a VL sample is defined by two cuts in distance we compute

$n(r)=\frac{\mathrm{d} N(r)}{\mathrm{d} r} \sim r^{D-1}$

i.e. the differential number counts in shells. We expect the exponent in Eq. (5) to be 2 when the distribution is uniform; in this case we also expect to see small (normalized) fluctuations generally decaying with volume or faster for super-homogeneous case (i.e. for standard cosmological density fields - see discussion in Gabrielli et al. 2004)

Results in the samples considered are shown in Figs. 5-8, where for each sample we have normalized the counts to the solid angle of the corresponding angular region. The best fit exponent (reported in the figures) fluctuates, and in several cases it is larger than 2. This means that there are large fluctuations as revealed by the non-smooth behaviors of $n(r)$ in the different samples. Similar evidence for the effect of large scale structures in these samples on other statistical quantities has been pointed out by Nichol et al. (2006).

This is a first rough indication that the question of uniformity at scales of order $100 \mathrm{Mpc} / h$ is not simple to resolve in these samples. These large fluctuations in slope and amplitude correspond to the presence of large scale galaxy structures extending up to the boundaries of the various samples considered. We do 


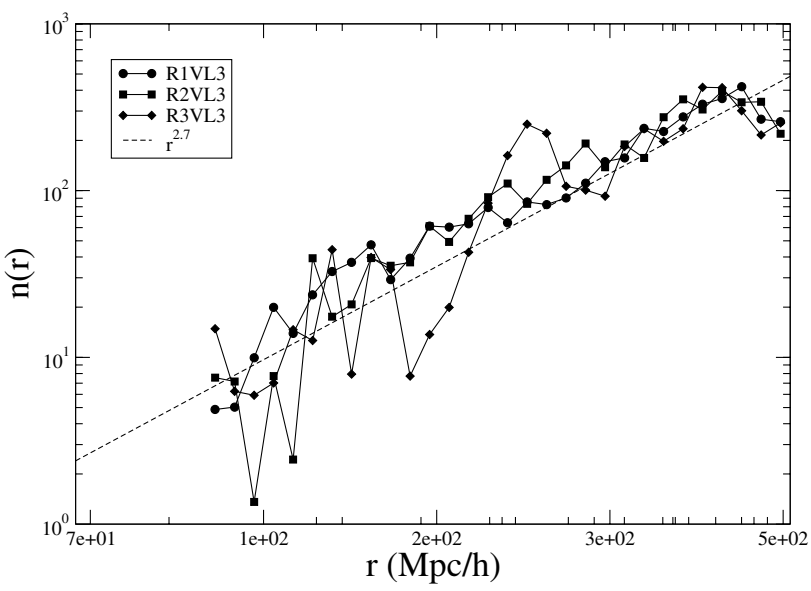

Fig. 7. The same as Fig. 5 but for the VL3 samples.

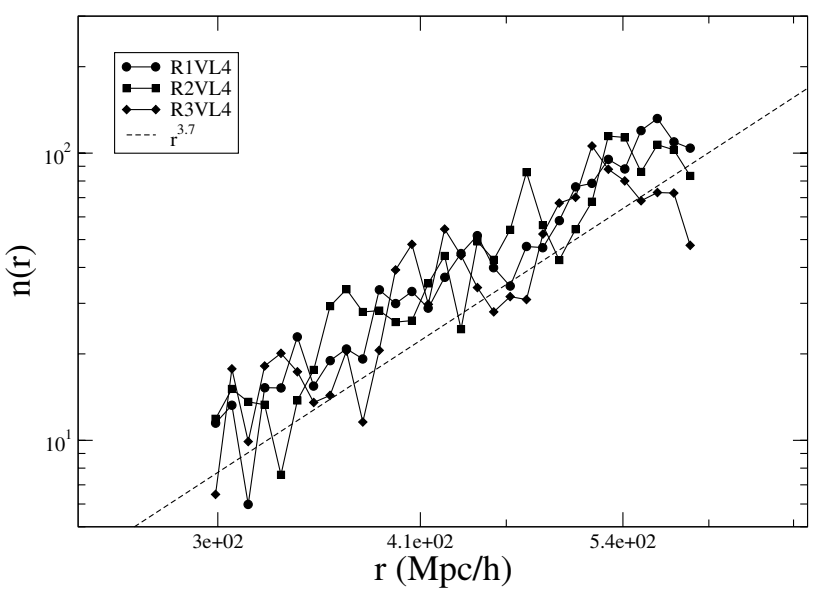

Fig. 8. The same as Fig. 5 but for the VL4 samples.

not present a more quantitative discussion of these behaviors as the statistics are rather weak.

\section{Correlation properties of galaxy distributions}

We now study the behavior of the conditional average density in the various VL samples discussed in the previous section. We use the full-shell estimator, discussed extensively in Gabrielli et al. (2004) and in Vasilyev et al. (2006). This estimator has the advantage of making no assumptions in the treatment of boundary conditions and it is the most conservative among estimators of two-pint correlations (see discussion in Kerscher 1999). The conditional density in spheres $\left\langle n(r)^{*}\right\rangle_{\mathrm{p}}$ is defined for an ensemble of realizations of a given point process, as

$\left\langle n(r)^{*}\right\rangle_{\mathrm{p}}=\frac{\langle N(r)\rangle_{\mathrm{p}}}{V(r)}$

This quantity measures the average number of points $\langle N(r)\rangle_{\mathrm{p}}$ contained in a sphere of volume $V(r)=\frac{4}{3} \pi r^{3}$ with the condition that the center of the sphere lies on an occupied point of the distribution (and $\langle\ldots\rangle_{\mathrm{p}}$ denotes the conditional ensemble average).

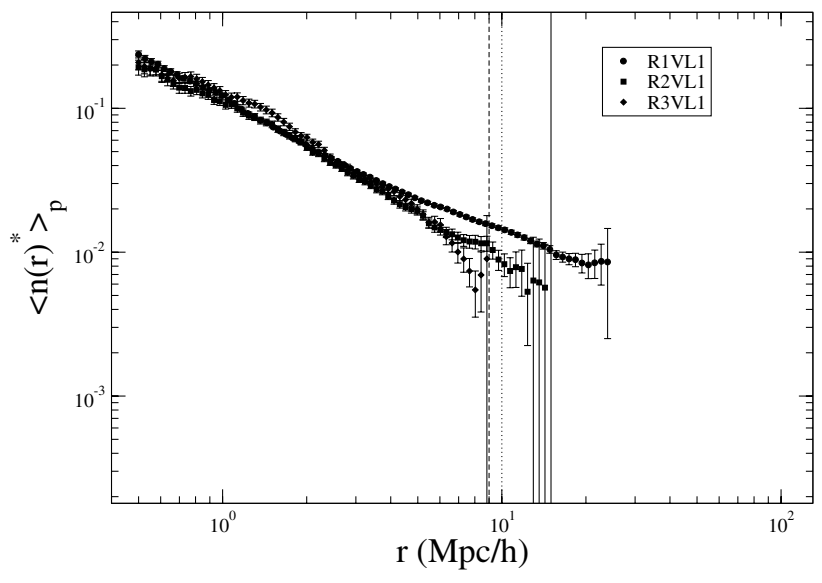

Fig. 9. Conditional density in spheres in the VL1 sample in the angular region R1, R2, R3. Here and in Figs. 10-12 we report, for each sample, a vertical line corresponding to the distance scale $R_{\mathrm{c}}$ discussed in Sect. 3.2 and shown in Table 4 (solid-line for R1, dotted-line for R2 and dashed-line for R3).

Such a quantity can be estimated ${ }^{1}$ in a finite sample by a volume average (supposing ergodicity of the point distribution)

$\left\langle n(r)^{*}\right\rangle_{\mathrm{p}}=\frac{1}{N_{\mathrm{c}}(r)} \sum_{i=1}^{N_{\mathrm{c}}(r)} \frac{N_{i}(r)}{V(r)}$,

where $N_{\mathrm{c}}(r)$ - the number of points chosen as centers of a sphere of radius $r$ fully contained in the sample volume - averaging by the sample points. (The estimation of the conditional density in shells $\langle n(r)\rangle_{\mathrm{p}}$ proceeds in the same way, except we consider spherical shells instead of spheres centered on the points - see e.g. Vasilyev et al. 2006).

This full-shell estimator has an important constraint: it is measured only in spherical volumes fully included in the sample volume. In this situation the number of centers $N_{\mathrm{c}}(r)$ over which the average Eq. (7) is performed becomes strongly dependent on the scale $r$ when $r \rightarrow R_{\mathrm{s}}, R_{\mathrm{S}}$ being the sample size. In this context such a length scale can be defined as the radius of the largest sphere fully included in the sample volume: the center of such a sphere lies in the middle of the sample volume.

Thus, when approaching the scale $R_{\mathrm{s}}$ there are two sources of fluctuations which increase the variance of the measurements. On the one hand the number of points over which the average is performed decreases very rapidly and on the other hand the remaining points are concentrated toward the center of the sample. In such a way systematic fluctuations may affect the estimation, given that these are not averaged out by the volume average. An estimation of the scale beyond which systematic effects become strong is thus important.

The following subsection discusses the measurements of $\left\langle n(r)^{*}\right\rangle_{\mathrm{p}}$ in the different VL samples, while Sect. 3.2 is devoted to the problem of the determination of the maximum scale up to which the volume average is properly performed, and thus beyond which systematic unaveraged fluctuations may affect the behavior of the conditional density.

1 For simplicity we use the same symbol for the ensemble average and for the estimator of all statistical quantities defined in this section. 


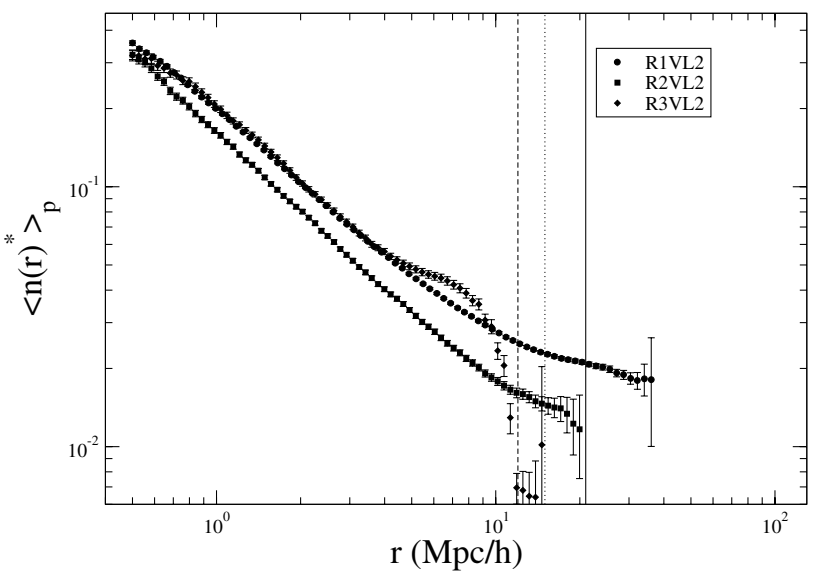

Fig. 10. As for Fig. 9 but for the VL2 samples.

\subsection{Estimation of the conditional density}

The results of the measurements in redshift space of the conditional density by the full-shell estimator, in VL samples with the same cuts in absolute magnitude and distance but in different angular regions, are reported in Figs. 9-12. The formal statistical error, reported in the figures, for the determination of $\left\langle n(r)^{*}\right\rangle_{\mathrm{p}}$ at each scale, can be derived from the dispersion of the average

$\Sigma^{2}(r)=\frac{1}{N_{\mathrm{c}}(r)} \sum_{i=1}^{N_{\mathrm{c}}(r)-1} \frac{\left(n(r)_{i}^{*}-\left\langle n(r)^{*}\right\rangle_{\mathrm{p}}\right)^{2}}{N_{\mathrm{c}}(r)-1}$

where $n(r)_{i}^{*}$ represents the determination from the $i^{\text {th }}$ point. Such an error is very small, except for the last few points. However, as discussed below, when $r \rightarrow R_{\mathrm{S}}$ systematic fluctuations can be more important than statistical ones.

One may note the following behaviors:

- In the three VL1 samples the signal is approximately the same up to $10 \mathrm{Mpc} / h$, where the conditional density has a power-law behavior

$\left\langle n(r)^{*}\right\rangle_{\mathrm{p}} \sim r^{-\gamma}$

with exponent $\gamma=1.0 \pm 0.1$. The sample R3VL1 has an $R_{\mathrm{S}}$ of the order of $10 \mathrm{Mpc} / h$, while the sample R1VL1 about $25 \mathrm{Mpc} / h$ and R2VL1 about $15 \mathrm{Mpc} / h$. In the two former samples the signal is different in the range of scale $10-20 \mathrm{Mpc} / h$ and is clearly affected by large systematic fluctuations.

- For the three VL2 samples the situation is similar to the previous one. There is a difference in the amplitude of R1VL2 and R2VL2 of about a factor of 2. Nevertheless the powerindex is very similar in all three samples and $\gamma=1.0 \pm 0.1$. All samples present a deviation from a power-law at their respective $R_{\mathrm{S}}=35,20,15 \mathrm{Mpc} / h$. These deviations are again a sign of finite size effects, reflecting systematic unaveraged fluctuations, as they occur at different scales in the three samples, but always at scales comparable to the sample size.

- For the case of VL3 samples the behavior of the conditional density is smoother at small scales: up to $30 \mathrm{Mpc} / h$ all the three samples present the same power-law correlation with an index $\gamma=1.0 \pm 0.1$. Thus the exponent is the same as in VL1 and VL2, but, given that $R_{\mathrm{s}}$ for these samples is larger than for VL1 and VL2, it extends to larger scales. The amplitude of the conditional density is almost the same in the three samples up to $\sim 30 \div 40 \mathrm{Mpc} / h$. Beyond such

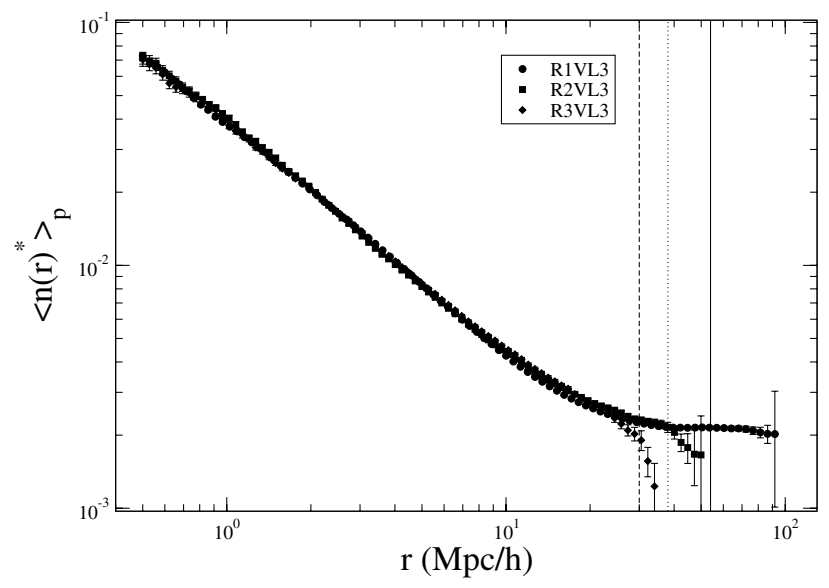

Fig. 11. As for Fig. 9 but for the VL3 samples.

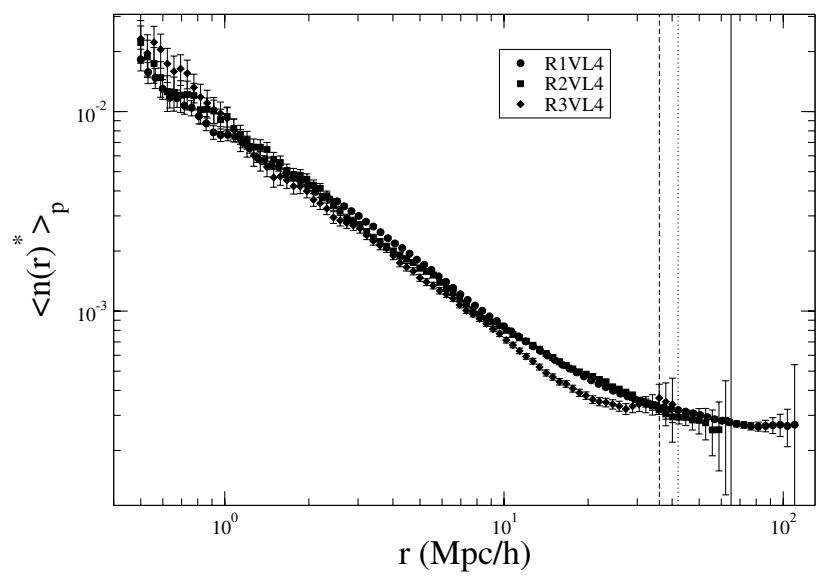

Fig. 12. As for Fig. 9 but for the VL4 samples.

a scale R1VL3 shows a flattened behavior, similar to the case R2VL3 although in this case there is a deviation at large scales (from about $40 \mathrm{Mpc} / h$ ). The sample size for R3VL3 is about $30 \mathrm{Mpc} / h$ and thus does not give any information on the larger scales. In the following section we present several tests to clarify whether the crossover to homogeneity which seems to be clear in the sample R1VL3 is stable in different samples and whether systematic fluctuations are negligible.

- The sample VL4 is the deepest one and the behavior measured is similar to VL3 although there is a clear difference at large scales and fluctuations are more evident. Up to $30 \mathrm{Mpc} / h$ the exponent is again $\gamma=1.0 \pm 0.1$, i.e. like VL1 and VL2 at smaller scales, and VL3 at the same scales.

The difference in amplitude of the conditional density in the different samples VL1, VL2 and VL3 is simply explained by considering the effect of the luminosity function in the selection of the galaxies (see Gabrielli et al. 2004, for a detailed treatment of this point).

Thus the correlation properties are independent of galaxy luminosity and they are characterized by a power-law index in the behavior of the conditional density $\gamma=1.0 \pm 0.1$ up to $30 \mathrm{Mpc} / h$. At larger scales, as shown for example in the two samples R1VL4 and R2VL4 the situation is less clear: fluctuations are more important because they are not smoothed out by the volume average. In the next subsection we define the range where the volume average is properly performed. 
Table 4. Main properties of the different subsamples considered in the $\mathrm{R} 1$ region. The angular limits of the cuts in the intrinsic coordinates of the survey $\eta$ and $\lambda$ (in degrees). The last column gives the number of points in the sample.

\begin{tabular}{cccccc}
\hline \hline Region name & $\eta_{1}$ & $\eta_{2}$ & $\lambda_{1}$ & $\lambda_{2}$ & $N$ \\
\hline R1_1VL1 & 9.0 & 22.5 & -47.0 & 8.0 & 1585 \\
R1_2VL1 & 22.5 & 36.0 & -47.0 & 8.0 & 1545 \\
R1_1VL2 & 9.0 & 22.5 & -47.0 & 8.0 & 7684 \\
R1_2VL2 & 22.5 & 36.0 & -47.0 & 8.0 & 7497 \\
R1_1VL3 & 9.0 & 22.5 & -47.0 & 8.0 & 13982 \\
R1_2VL3 & 22.5 & 36.0 & -47.0 & 8.0 & 13993 \\
R1_1VL4 & 9.0 & 22.5 & -47.0 & 8.0 & 3343 \\
R1_2VL4 & 22.5 & 36.0 & -47.0 & 8.0 & 3399 \\
\hline
\end{tabular}

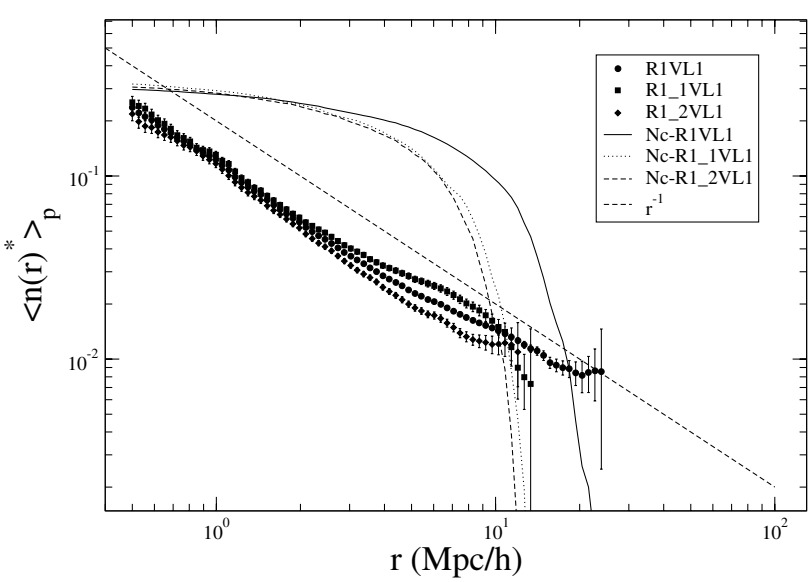

Fig. 13. Conditional density in spheres in the R1VL1 sample and in the 2 subsamples defined by the angular cut performed as discussed in the text. The lines labeled with $N_{\mathrm{c}}$ represent the behavior of the number of centers used in the average (Eq. (7)) arbitrarily normalized.

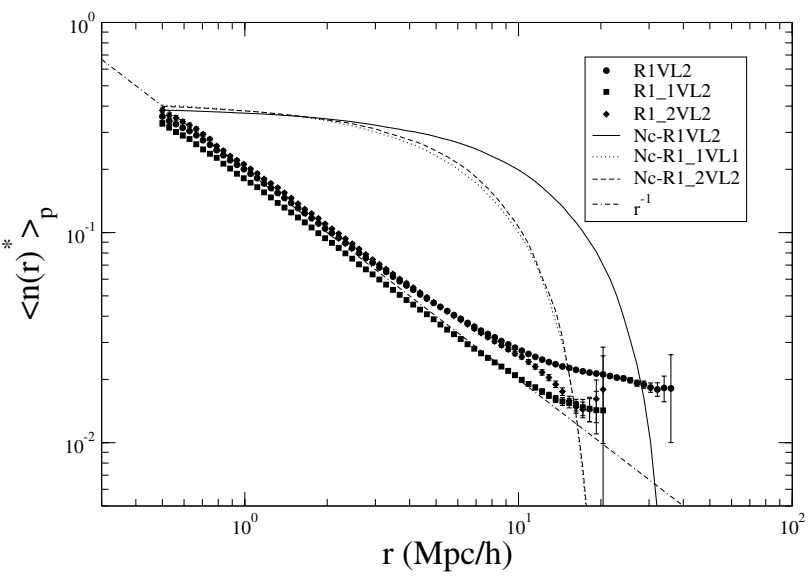

Fig. 14. As Fig. 13 but for the R1VL2 sample.

\subsection{Finite volume effects}

In order to quantify the finite volume effects previously mentioned, we have divided each of the VL samples of the R1 field into two non-overlapping contiguous angular regions, and we have recomputed the conditional density in each of the $2 \times$ 4 samples. The properties of these subsamples are listed in Table 4. In Figs. 13-16 we show the results.

As already mentioned the average computed by Eq. (7) is made by changing, at each scale $r$, the number $N_{\mathrm{c}}(r)$ of points which contribute. This scale-dependency follows from the requirement that only those points are chosen for which, when

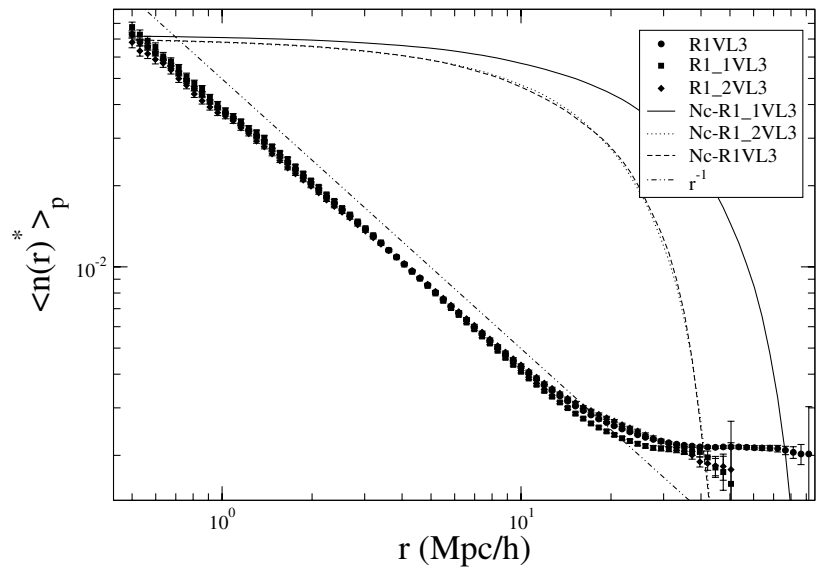

Fig. 15. As Fig. 13 but for the R1VL3 sample.

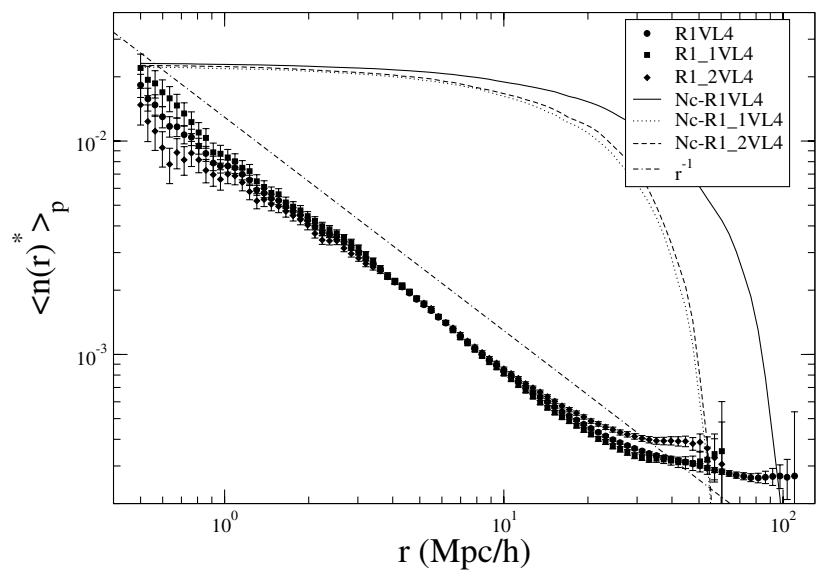

Fig. 16. As Fig. 13 but for the R1VL4 sample.

chosen as centers of a sphere of radius $r$, the volume does not overlap or intersect the boundaries of the sample. In this way, in a sample of size $R_{\mathrm{s}}$, when $r \ll R_{\mathrm{S}}$ almost all points will contribute to the average, while when $r \rightarrow R_{\mathrm{s}}$ only those points lying close to the center of the volume will be taken into account in the average. Hence at large scales the average is performed on a number of points that exponentially decay when $r \rightarrow R_{\mathrm{S}}$. In Figs. 13-16 we show the behavior of the number of centers $N_{\mathrm{c}}(r)$ as a function of scale, normalized to an arbitrary factor. The normalization is simple because at small scales $N_{\mathrm{c}}(r)=N$ where $N$ is the number of points contained in a given VL sample: at such small scales all points contribute to the statistics. At a scale comparable with, but smaller than, the sample size there is an abrupt decay of this quantity: this means that only few points contribute to the average at large scales.

That systematic fluctuations are more important than statistical ones can be noticed from the behavior of the conditional density in Figs. 13-16 by comparing the behaviors in the original sample (e.g. R1VL1) and in the two separate subsamples (e.g. R1_1VL1 and R1_2VL1). When the distance scale approaches the boundaries of the samples there are systematic variations that are larger than the (small) error bars derived from Eq. (8). As already mentioned, in some cases there is evidence for a more flatter behavior while in other cases instead the conditional density show a decay up to the sample boundaries which is slower than at smaller scales. This situation suggests caution in the interpretation of the large scale tail of the conditional density. The question 


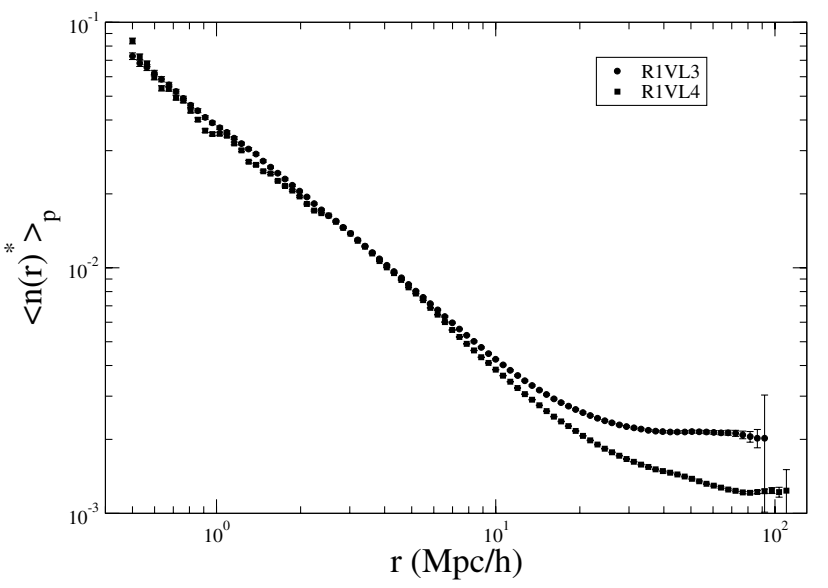

Fig. 17. Conditional density in spheres in the R1VL3 and R1VL4 samples, normalized to have the same amplitude at $1 \mathrm{Mpc} / h$. The large scale behavior $(r>30 \mathrm{Mpc} / h)$ is different due to the effect of systematic fluctuations.

is how to quantify the regime where systematic fluctuations are important and may affect the behavior of the conditional density.

One may define a criterion for the statistical robustness of the volume average by imposing for example that $N_{\mathrm{c}}(r)$ must be larger than a certain value. While this can certainly give a useful indication, the problem of the volume average is more subtle. In fact when $r \rightarrow R_{\mathrm{S}}$ there can be sufficient points for $N_{\mathrm{c}}(r)$ to be larger than a given pre-defined value: however it may happen that all these points lie, for example, in a cluster located close to the sample center. In this situation the volume average is not properly performed, in the sense that all points "see" almost the same volume.

A way to clarify such a situation has been proposed by Joyce et al. (1999). One may compute the average distance between the $N_{\mathrm{c}}(r)$ centers at the scale $r$ :

$$
R_{\mathrm{c}}(r)=\frac{1}{N_{\mathrm{c}}(r)\left(N_{\mathrm{c}}(r)-1\right)} \sum_{i, j=1}^{N_{\mathrm{c}}(r)}\left|\boldsymbol{r}_{i}-\boldsymbol{r}_{j}\right|
$$

where $\boldsymbol{r}_{i}$ and $\boldsymbol{r}_{j}$ are two of the $N_{\mathrm{c}}(r)$ points. A criterion for statistical validity of the volume average is then

$R_{\mathrm{c}} \geq 2 \times r$

which implies that the average distance between sphere centers is larger than twice the scale at which the conditional density is computed, assuring in this way the independence of the different terms in the average. The values of $R_{\mathrm{c}}$ for the different samples is reported in Table 3 and this length-scale is indicated as a vertical line in Figs. 9-12. In practice all samples show an $R_{\mathrm{c}}$ of less than $40 \mathrm{Mpc} / h$ with the exception of R1VL3 and R1VL4 for which $R_{\mathrm{c}}=54$ and $65 ; \mathrm{Mpc} / h$ respectively. However, in these two samples the conditional density does behave differently at large scales (see Fig. 17), in the sense that the change of slope occurs at different scales and thus at a different average density value. Thus it is very hard to conclude about the correlation properties at such large scales.

However we note that there is enough evidence that the signal is smoother on scales $>40 \mathrm{Mpc} / h$ and that sample-tosample fluctuations or the variations in radial counts (discussed in Sect. 2) are smaller, thus indicating a tendency toward a more uniform distribution. However these data do not unambiguously support a clear evidence in favor of homogeneity at scales of the order of $70 \mathrm{Mpc} / h$, as Hogg et al. (2005) found by analyzing the

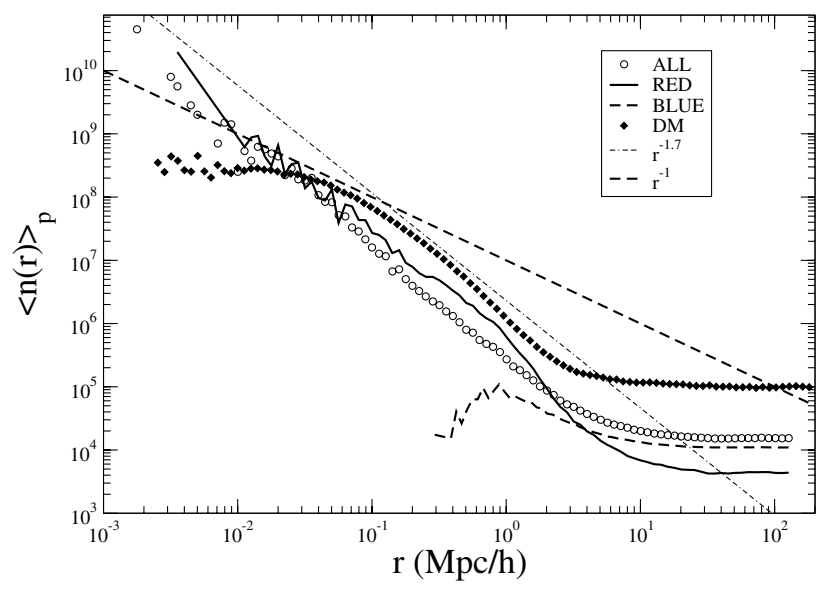

Fig. 18. Conditional density for the four samples of points selected in the simulation: the original dark matter (DM) field, all "galaxies" (ALL), blue galaxies (BLUE) and red galaxies (RED). The conditional density for dark matter particles (DM) has been normalized arbitrarily. The reference dashed-dotted line has a slope $\gamma=1.7$. The dashed line with $\gamma=1$, corresponding to the slope measured in the galaxy samples is also reported.

LRG sample, because the change in correlation properties occurs at scales comparable to the scales $R_{\mathrm{s}}$ and $R_{\mathrm{c}}$. We conclude that these data support a change of slope, with a clear tendency for $\gamma<1$, but with an undefined value.

These tests indicate that the availability of larger samples, provided, for example, by DR5, will allow one to understand these systematic variations. To study scales of the order of $100 \mathrm{Mpc} / h$, samples with $R_{\mathrm{s}} \approx 300 \mathrm{Mpc} / h$ are needed. However the full SDSS data will provide us with such large and complete catalogs.

\section{Correlation properties of cosmological $\mathbf{N}$-body simulations}

Gravitational clustering in the regime of strong fluctuations is usually studied through gravitational $N$-body simulations. The particles are not meant to describe galaxies but collisionless dark-matter mass tracers. During gravitational evolution complex non-linear dynamics make non-linear structures at small scales, while at large scales a linear amplification occurs according to linear perturbation theory. Thus, while on large scales correlation properties do not change from the beginning - except a simple linear scaling of amplitudes - at small scales nonlinear correlations occur. Typically in these simulations nonlinear clustering is formed up to scales of the order of a few Mpc.

At late times one can identify subsamples of points that trace the high density regions, and these would represent the sites for galaxy formation, whose statistical properties are ultimately compared with the ones found in galaxy samples.

In order to study this problem we consider the GIF galaxy catalog (Kauffmann et al. 1999) constructed from a $\Lambda$ CDM simulation run by the Virgo consortium (Jenkins et al. 1998). This is done firstly identifying the halos, which represent almost spherical structures with a power-law density profile from their center. The number of galaxies belonging to each halo is set proportional to the total number of points belonging to the halo to a certain power. This procedure identifies points lying in high density regions of the dark-matter particles. One may assign to each point a luminosity and a color on the basis of a certain 


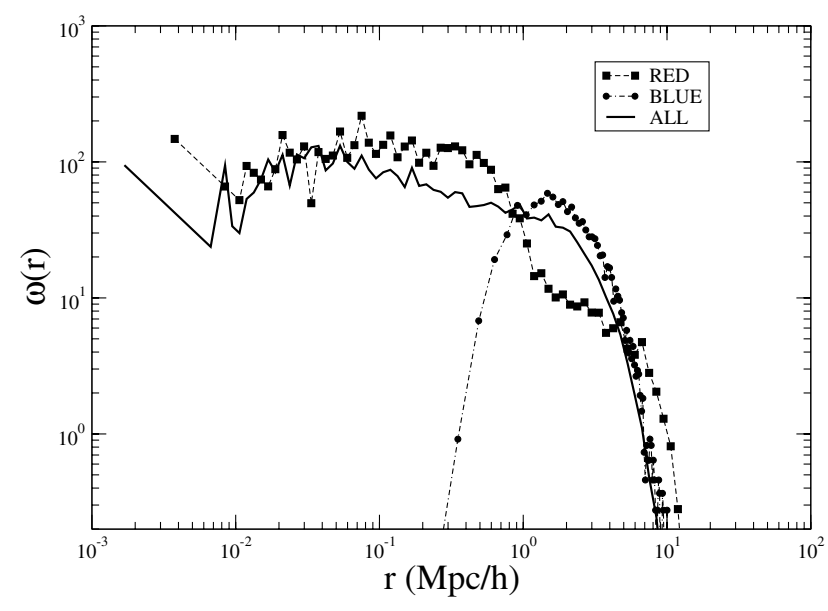

Fig. 19. Nearest neighbors probability distribution for three point sets selected in the simulation (see discussion in the text): all "galaxies" (ALL), blue galaxies (BLUE) and red galaxies (RED).

criterion which is not relevant for what follows (see Sheth et al. 2001, and reference therein). The resulting catalog is divided into two subsamples based on "galaxy" color $B-I$ as in Sheth et al. (2001): (brighter) red galaxies (for which $B-I$ is redder than 1.8) and (fainter) blue galaxies ( $B-I$ bluer than 1.8).

In summary four samples of points may be considered: (i) the original dark matter particles with $N=256^{3}$ particles; (ii) all galaxies with $N=15445$; (iii) blue galaxies with $N=11023$; and (iv) red galaxies with $N=4422$.

In order to understand the correlation properties in the sampled point distributions it is useful to study the behavior of the conditional density which, as already discussed, has a straightforward interpretation in terms of correlations; results are shown in Fig. 18. The red galaxies are responsible for the strong correlations observed in the full sample as the conditional density is almost the same as for all galaxies at small scales. At large scales there is instead a fast decrease as the sample average of red galaxies is smaller than the one of all galaxies (there are fewer objects). For red galaxies the sampling is local, i.e. their conditional density is (almost) invariant at small scales. Clearly, as there are globally less objects, the sample density of red galaxies is smaller than that of all galaxies. On the other hand blue galaxies present only some residual correlations at small scales, and they are more numerous than red galaxies.

The small scale properties of these distributions can be studied by analyzing the NN probability distribution (see Fig. 19). Blue galaxies have a bell-shaped distribution, typical of the case where correlation are very weak. Instead red and all galaxies present almost the same function, with a long small-scale tail, which is a typical feature indicating the presence of strong twopoint correlations (see discussion in Baertschiger \& Sylos Labini 2002). This situation is different from the one detected in the samples of DR4 as shown in Figs. 1-4, where the NN probability distribution has the same shape for all samples considered.

The main points are the following:

- The slope of the conditional density in all the artificial samples considered here is different from $\gamma=1.0 \pm 0.1$ measured in the real galaxy data. In particular for those mock samples (red galaxies, all galaxies and dark matter particles) where correlations are power-law, the slope is $\gamma=1.7 \pm 0.1$ in the range $[0.01,5] \mathrm{Mpc} / h$ while a clear transition toward homogeneity occurs at scales of the order of $10 \mathrm{Mpc} / h$. These different slopes can be explained by the fact that we compare a measure in redshift space, in the case of real data, which can be affected by redshift distortions, with the mock catalogs where the conditional density has been measured in real space. We will examine this point in more detail in a forthcoming paper.

- Small scale properties, as detected by the NN probability distribution, are different in the real and artificial samples.

- The conditional densities of mock blue and red galaxies are different at all scales and blue galaxies show almost no correlations.

- Both mock red and blue galaxies show a well-defined transition to homogeneity at a scale of the order of $10 \mathrm{Mpc} / h$. As we have already mentioned, this is not the behavior observed in the data. Particularly the range of non-linear structures seem to be much wider in the real data than in the simulations.

While the comparison between correlation properties of real galaxies and mock galaxy catalogs constructed from points selected in $N$-body simulations is usually performed by the analysis of the reduced two-point correlation function, here we have presented the comparison of the conditional density and of the NN probability distributions. We find that some important disagreements between data and simulations are evident when the behavior of these statistical quantities are considered. This is not the same conclusion that one may reach by analyzing the reduced correlation function $\xi(r)$ : the reason is that in the estimation of $\xi(r)$ one uses the estimation of the sample average, which introduces a finite-size effect which may affect both the amplitude and slope of this function (see e.g. Gabrielli et al. 2004, for a detailed discussion of this point). The estimation of the conditional density is less affected by finite-volume effects and the comparison between different sample is straightforward.

Note that the data are analyzed in redshift space and the simulations in real space. However given that velocities are typically smaller than $500 \mathrm{~km} \mathrm{~s}^{-1}$ the difference between real and redshift space cannot be accounted by the effects of peculiar velocities on scales larger than $5 \mathrm{Mpc} / h$. The problem of the relation between real and redshift space, considering the finite size effects present when strong correlations characterize the data, has been discussed in Vasilyev et al. (2006).

\section{Discussion and conclusions}

Our main results are the following:

(i) In all VL samples we find that in the range of scales $0.5 \leq$ $r \lesssim 30 \mathrm{Mpc} / h$ the conditional density shows a power-law correlation with a power-law index $\gamma=1.0 \pm 0.1$. This result is in good agreement with the behavior found in other smaller samples by Sylos Labini et al. (1998), Joyce et al. (1999) and in the SDSS LRG sample by Hogg et al. (2005), and with the correlation properties measured by Vasilyev et al. (2006) in the 2dFGRS.

We do not confirm the results of Zehavi et al. (2004) who found a departure from a power-law in the galaxy correlation function at a scale of the order of $1 \mathrm{Mpc} / h$ : their analysis was performed in real space while ours is in redshift space. In this range of scale nearest-neighbor correlations dominate the behavior of the conditional density and thus also of the reduced correlation function and for a detailed understanding of this regime a study of the nearestneighbor is necessary.

We do not find a luminosity or color dependence of the galaxy the conditional density in the regime of strongly 
non-linear correlations. In this respect Zehavi et al. (2005) have considered the behavior of the reduced two-point correlation function, and concluded that there is a color (luminosity) dependence of galaxy correlations. This apparent disagreement can be understood by considering that the reduced two-point correlation function can be strongly affected by finite-size effects in the regime where the conditional density presents power-law correlations (see discussion, e.g., in Joyce et al. 2005). Moreover results by Zehavi et al. (2005) have been obtained in real space: in Vasilyev et al. (2006) we discussed the kind of finite size effects which perturb the estimation of $\xi(r)$ when the conditional density has power-law correlations.

(ii) In the range $30 \lesssim r \lesssim 100 \mathrm{Mpc} / h$ the situation is less clear: as we discussed, finite volume effects are important in this range of scales and systematic unaveraged fluctuations may affect the results. We have presented several tests to show the role of finite volume effects and to determine the range of scales where they perturb the estimation of the conditional density, finding that in all but two samples the volume average is properly performed up to $R_{\mathrm{c}} \approx 40 \mathrm{Mpc} / h$. In the remaining two samples we have shown that systematic fluctuations persist up to their boundaries $R_{\mathrm{s}}$.

Thus in the range $30 \lesssim r \lesssim 100 \mathrm{Mpc} / h$ we find evidence for a more uniform distribution and hence a smaller power law index $(\gamma<1)$ in the conditional density. This is a stable result in all samples considered. However a detailed analysis of the behavior of the conditional density in all samples does not allow us to conclude either that there is definitive crossover to homogeneity at a scales of order $70 \mathrm{Mpc} / \mathrm{h}$ as Hogg et al. (2005) have concluded by considering the LRG sample, or that there is a change of power-law index beyond $30 \mathrm{Mpc} / h$ which remains stable up to the samples limit, i.e. up to $100 \mathrm{Mpc} / h$. Both possibilities are still open and will be clarified by forthcoming data releases of SDSS as the solid angle will increase.

(iii) The comparison of mock galaxy catalogs constructed from particle distributions extracted from cosmological $N$-body simulations with real galaxy data reveals a problematic situation. On the one hand we have discussed the fact that the slope of the conditional density is different from the one measured in real catalogs. On the other hand we have also stressed that when constructing artificial galaxy samples from dark matter particles in $N$-body simulations, there are different behaviors in the conditional density according to the different selection criteria used, and thus on the different way to assign "luminosity" and "color" to the artificial galaxies. This behavior is not in agreement with the data, as in all samples analyzed here, the same slope in the conditional density is measured. The same situation is present when the NN probability distribution is considered. Then in $N$-body simulations, structures are smaller than in real data, as shown by the definitive crossover to homogeneity at about $10 \mathrm{Mpc} / h$ found in the $N$-body particle distribution, contrary to the galaxy case where the crossover may happen on much larger scales of the order of $100 \mathrm{Mpc} / h$.

We have used a very conservative statistical analysis which introduces important constraints on the way we treat the data. For example if the distribution would have been uniform on scales smaller than the actual sample sizes, the conditional density estimation could done for all points in the sample, even on large scales, not just the points near the center of the sample, because it can be assumed that the volume outside the survey region is statistically similar to the volume inside. This is the standard approach with conventional two-point statistics in the literature. On the other hand we have used, for example, periodic boundary conditions in the analysis of artificial simulations, as in this case the distribution is periodic beyond the simulation box, by construction. However, as we do not know whether this is the case for the galaxy distribution, and because we would like to test this point, we have used more conservative statistics to analyze the real data. This, instead of being a limitation, allows us to derive results about galaxy correlation properties that are unbiased by finite size effects. Indeed, when using less conservative methods, one is implicitly making the assumption that finite size effects, induced by long-range correlations in the galaxy distribution, are negligible. Here we instead test that this is the case in the data we consider and we find evidence that, because of the long-range nature of galaxy correlations, there are subtle finite size effects which should then be a serious warning to the use of less conservative statistical methods. Having used more conservative statistics we are able to obtain results that are less biased by finite size effects (which ultimately appear from the presence of large fluctuations represented by large scale structures) than the ones derived by a statistical analysis which makes use of some untested assumptions to derive its results. For example we find that the exponent of the conditional density is -1 instead of -1.7 as derived with a more "relaxed" analysis, at the same scales. The measurements of the conditional density has been performed in real space in the mock catalogs and in redshift space in the real samples, and this can be the origin of the different values of the correlation exponents. Whether this is case, or a finite size effect plays a crucial role will be studied in a forthcoming paper.

We discuss our results in relation to theoretical models of fluctuations in standard cosmologies. It has been shown (see e.g. Gabrielli et al. 2004) that the only feature of the primordial correlations, defined in theoretical models like the cold dark matter (CDM) one, that can be detected in galaxy data is represented by the large scale tail of the reduced correlation function. In terms of correlation function $\xi(r), \mathrm{CDM}$ models presents the following behavior: it is positive at small scales, it crosses zero at a certain scale and then it is negative approaching zero with a tail which goes as $r^{-4}$ in the region corresponding to $P(k) \sim k$ (see e.g. Gabrielli et al. 2004). The super-homogeneity (or HarrisonZeldovich) condition says that the volume integral over all space of the correlation function is zero

$\int_{0}^{\infty} d^{3} r \xi(r)=0$.

This means that there is a fine balance between small-scale positive correlations and large-scale negative anti-correlations. This is the behavior that one would like to detect in the data in order to confirm inflationary models. Up to now this search has been done through the analysis of the galaxy power spectrum (PS) which should scale as $P(k) \sim k$ at small $k$ (large scales). No observational test of this behavior has been provided yet. However for this case one should consider an additional complication.

In standard models of structure formation, galaxies result from a sampling of the underlying CDM density field: for instance one selects only the highest fluctuations of the field that would represent the locations where a galaxy will eventually form. It has been shown that sampling a super-homogeneous fluctuation field changes the nature of the correlations (Durrer et al. 2003). The reason for this can be found in the property of super-homogeneity of such a distribution: the sampling necessarily destroys the surface nature of the fluctuations, as it introduces a volume (Poisson-like) term in the mass fluctuations, 
giving rise to a Poisson-like PS on large scales $P(k) \sim$ const. The "primordial" form of the PS is thus not apparent in that which one would expect to measure from objects selected in this way. This conclusion should hold for any generic model of bias and its size has to be established in any given model (Durrer et al. 2003).

On the other hand, one may show (Durrer et al. 2003) that the negative $r^{-4}$ tail in the correlation function does not change with sampling: on large enough scales, where in these models (anti) correlations are small enough, the biased fluctuation field has a correlation function that is linearly amplified with respect to the underlying dark matter correlation function. For this reason the detection of such a negative tail would be the main confirmation of models of a primordial density field. This will be possible if firstly a clear determination of the homogeneity scale is obtained, and then if the data are statistically robust enough to allow the determination of the correlation when it is $\xi(r) \ll 1$. While Eiseinstein et al. (2005) claimed to have measured that $\xi(r) \approx 0.01$ at scales of order $100 \mathrm{Mpc} / h$ in a sample of SDSS LRG galaxies, here we cannot confirm these results as our analysis does not extend to such large scales with robust statistics. However from the large fluctuations observed, for example in the behavior of the radial counts and in sample-tosample variations of the conditional density at such large scales, we conclude that this result needs more studies, and perhaps much larger samples, to be confirmed.

Acknowledgements. We thank Andrea Gabrielli, Michael Joyce and Luciano Pietronero for useful discussions and comments. Yu.V.B. and N.L.V. thank the "Istituto dei Sistemi Complessi" (CNR, Rome, Italy) for the kind hospitality during the writing of this paper. FSL acknowledges the EC grant No. 517588 "Statistical Physics for Cosmic Structures" and the MIUR-PRIN05 project on "Dynamics and Thermodynamics of systems with long range interactions" for financial supports. Yu.V.B and N.L.V acknowledge the partial financial support by Russian Federation grants NSh-8542.2006.2 and RNP.2.1.1.2852.

\section{References}

Adelman-McCarthy, J. K., et al. 2005 [arXiv: astro-ph/0507711] Baryshev, Yu., \& Teerikorpi, P. 2005, Bull. Special Astrophys. Obs, 59, 146 Baertschiger, T., \& Sylos Labini, F. 2004, Phys. Rev. D, 69, 123001-1 Blanton, M. R., Dalcanton, J., Eisenstein, D., et al. 2001, AJ, 121, 2358 Durrer, R., Gabrielli, A., Joyce, M., \& Sylos Labini, F. 2003, ApJ, 585, L1 Eisenstein, D. J., Zehavi, I., Hogg, D. W., et al. 2005, ApJ, 633, 560

Gabrielli, A., Sylos Labini, F., Joyce, M., \& Pietronero, L. 2004, Statistical physics for cosmic structures, Springer Verlag

Kerscher, M. 1999, A\&A, 343, 333

Hogg, D. W., Eistenstein, D. J., Blanton, M. R., et al. 2005, ApJ, 624, 54 Kauffmann, G., Colberg, J., Diaferio, A., \& White, S. D. M. 1999, MNARS, 303, 188

Jenkins, A., Frenk, C. S., Pearce, F. R., et al. 1998, ApJ, 499, 20

Joyce, M., Montuori, M., \& Sylos Labini, F. 1999, ApJ, 514, L5

Joyce, M., Sylos Labini, F., Gabrielli, A., Montuori, M., \& Pietronero, L. 2005, A\&A, 443, 11

Nichol, R. C., Sheth, R. K., Suto, Y., et al. 2006 [arXiv: astro-ph/0602548]

Pietronero, L. 1987, Physica A, 144, 257

Sheth, R. K., Diaferio, A., Hui, L., \& Scoccimarro, R. 2001, MNRAS, 326, 463 Strauss, M. A., Weinberg, D. H., Lupton, R. H., et al. 2002, AJ, 124, 1810 Sylos Labini, F., Montuori, M., \& Pietronero, L. 1998, Phys. Rep., 293, 66 Vasilyev, N. L., Baryshev, Yu. V., \& Sylos Labini, F. 2006, A\&A, 447, 431 Zehavi, I., Weinberg, D. H., Zheng, Z., et al. 2004, ApJ, 608, 16

Zehavi, I., Zheng, Z., Weinberg, D. H., et al. 2005, ApJ, 630, 1 